\title{
Other Incorrect Electrode Placement
}

National Cancer Institute

\section{Source}

National Cancer Institute. Other Incorrect Electrode Placement. NCI Thesaurus. Code C114176.

An electrocardiographic recording in which one or more electrodes are incorrectly placed but further details are not provided. (CDISC) 